\title{
LA IMPORTANCIA DE LA LECTURA Y SU PROBLEMÁTICA EN EL CONTEXTO EDUCATIVO UNIVERSITARIO. EL CASO DE LA UNIVERSIDAD JUÁREZ AUTÓNOMA DE TABASCO (MÉXICO)
}

\author{
Ariel Gutiérrez Valencia y Roberto Montes de Oca García \\ Universidad Juárez Autónoma de Tabasco, México
}

\section{DEFINICIÓN DE LA LECTURA}

Actualmente existe una gran diversidad de definiciones en torno a la lectura que son múltiples y acertadas, ya que en cada una de ellas se contemplan una serie de categorías conceptuales que ofrecen diferentes aspectos sobre esta capacidad eminentemente humana, y que permiten su análisis en toda su complejidad.

En este artículo se reconoce a la lectura " Como un proceso interactivo de comunicación en el que se establece una relación entre el texto y el lector, quien al procesarlo como lenguaje e interiorizarlo, construye su propio significado. En este ámbito, la lectura se constituye en un proceso constructivo al reconocerse que el significado no es una propiedad del texto, sino que el lector lo construye mediante un proceso de transacción flexible en el que conforme va leyendo, le va otorgando sentido particular al texto según sus conocimientos y experiencias en un determinado contexto." ${ }^{1}$

Desde esta perspectiva, el acto de leer se convierte en una capacidad compleja, superior y exclusiva del ser humano en la que se comprometen todas sus facultades simultáneamente y que comporta una serie de procesos biológicos, psicológicos, afectivos y sociales que lo llevan a establecer una relación de significado particular con lo leído y de este modo, esta interacción lo lleva a una nueva adquisición cognoscitiva. $^{2}$

Es importante señalar que la concepción de lectura que se postula en este artículo contraria a la tradicional, pone énfasis en la actividad que despliega el lector y reconoce su papel activo para construir el significado del texto. Desde esta concepción constructivista, la lectura se convierte en una actividad eminentemente social y fundamental para conocer, comprender, consolidar, analizar, sintetizar, aplicar, criticar, construir y reconstruir los nuevos saberes de la humanidad y en una forma de aprendizaje importante para que el ser humano se forme una visión del mundo y se apropie de él y el enriquecimiento que le provee, dándole su propio significado.

Después de haber señalado con claridad nuestro concepto de lectura, analizaremos a continuación cuales sonsus exigencias y su problemática en el contexto educativo universitario desde una perspectiva globalizada al amparo de los nuevos paradigmas mundiales, con especial referencia a la Universidad Juárez Autónoma de Tabasco.

\footnotetext{
${ }^{1}$ Margarita Gómez Palacios y otros. La lectura en la escuela. México : SEP, 1996. pp. 19-20

${ }^{2}$ Ana Arenzana y Aureliano García. Espacios de lectura : estrategias metodológicas para la formación de lectores. México : FONCA, 1995. p. 17
} 


\section{LA LECTURA EN EL CONTEXTO INTERNACIONAL}

Hoy día vivimos en un mundo globalizado habitado por 6,200 millones de personas, de las cuales, de acuerdo con la UNESCO, solamente 1,155 millones tienen acceso a una educación formal en sus diferentes grados, niveles y modalidades; mientras que en contraste, 876 millones de jóvenes y adultos son considerados analfabetos y 113 millones de niños en edad escolar se encuentran fuera de las aulas de las escuelas por diversas circunstancias. ${ }^{3}$

Ante este panorama mundial caracterizado por la pobreza extrema, la inequidad y la falta de oportunidades para todos para acceder a una educación digna para aspirar a una vida mejor, diversos organismos internacionales como la OCDE, la UNESCO, el BID, el Banco Mundial y la CEPAL han señalado que en los nuevos escenarios mundiales dominados por la globalización, la competitividad, la alta tecnología y la información, la educación y la lectura se constituyen en los pilares estratégicos del desarrollo de las naciones y por consiguiente, en una mejor posibilidad de aspirar a una vida mejor por parte de los ciudadanos.

A este respecto, la Organización para la Cooperación y el Desarrollo Económico (OCDE) ha manifestado que la lectura en especial debe ser considerada prioritariamente por todos sus países miembros como un indicador importante del desarrollo humano de sus habitantes. Al hacer referencia a este aspecto, la OCDE ha señalado recientemente que"EI concepto de capacidad o competencia lectora retomada por muchos países hoy en día, es un concepto que es mucho más amplio que la noción tradicional de la capacidad de leer y escribir (alfabetización), en este sentido, señala la OCDE la formación lectora de los individuos para una efectiva participación en la sociedad moderna requiere de la habilidad para decodificar el texto, interpretar el significado de las palabras y estructuras gramaticales, así como construir el significado. También implica la habilidad para leer entre líneas y reflexionar sobre los propósitos y audiencias a quien se dirigen los textos. La capacidad lectora involucra por tanto, la habilidad de comprender e interpretar una amplia variedad de tipos de texto y así dar sentido a lo leído al relacionarlo con los contextos en que aparecen. En síntesis, la capacidad lectora consiste en la comprensión, el empleo y la reflexión a partir de textos escritos y virtuales, con el fin de alcanzar las metas propias, desarrollar el conocimiento y el potencial personal y participar en la sociedad " 4

Asimismo, al referirse a la importancia de la lectura en el contexto de los nuevos paradigmas mundiales este organismo multilateral ha especificado que"Las actuales circunstanciasestán obligando a los individuos en todo el planeta a reflexionar sobre el contenido de un texto conectando la información encontrada en dicha fuente con el conocimiento obtenido de otros textos, de tal suerte que los lectores deben evaluar las afirmaciones realizadas en el texto frente a su propio conocimiento del mundo... Los lectores deben en los nuevos contextos, ser capaces de desarrollar una comprensión de lo que se dice y de lo que se intenta en un texto, y deben contrastar la representación mental derivada del texto frente a lo que sabe y cree, bien sobre la base de información previa, bien sobre la base de información encontrada en

\footnotetext{
${ }^{3}$ UNESCO. World Education Report, 2000. París : UNESCO, 2002. p. 54-60

${ }^{4}$ OECD. Programme for International Student Assessment. Reading, mathematical and scientific literacy. París : OECD. 2000. pp.12-19
} 
otros textos, utilizando tanto conocimientos generales como específico, así como la capacidad de razonamiento abstracto. " 5

A fin de revalizar la capacidad de la lectura en el desarrollo de los seres humanos en el contexto mundial, la Organización para la Cooperación y el Desarrollo Económico recientemente ha emprendido una amplia investigación para diagnosticar la problemática de la lectura entre los estudiantes de sus países miembros. Las conclusiones de estas investigaciones han sido publicadas en el documento denominado "Programme for International Student Assessment. Sample tasks from the PISA 2000. Assessment of reading, mathematical and scientific literacy"

A continuación se presenta un cuadro comparativo que nos muestra las capacidades de lectura de los estudiantes de 15 años en los países miembros de la OCDE, así como su rango de posiciones posibles.

\begin{tabular}{|l|c|c|c|}
\multicolumn{1}{c}{ País } & $\begin{array}{c}\text { Desempeño } \\
\text { Promedio } \\
\text { Puntaje }\end{array}$ & RANGO DE POSICIONES \\
\hline Finlandia & 546 & 1 & Baja \\
\hline Canadá & 534 & 2 & 4 \\
\hline Nueva Zelanda & 529 & 2 & 8 \\
\hline Australia & 528 & 2 & 9 \\
\hline Irlanda & 527 & 3 & 9 \\
\hline Corea & 525 & 4 & 9 \\
\hline Reino Unido & 523 & 5 & 9 \\
\hline Japón & 522 & 3 & 10 \\
\hline Suecia & 516 & 9 & 11 \\
\hline Austria & 507 & 11 & 16 \\
\hline Bélgica & 507 & 11 & 16 \\
\hline Islandia & 507 & 11 & 15 \\
\hline Noruega & 505 & 11 & 16 \\
\hline Francia & 505 & 11 & 16 \\
\hline Estados Unidos & 504 & 10 & 20 \\
\hline Dinamarca & 497 & 16 & 19 \\
\hline Suiza & 494 & 16 & 21 \\
\hline España & 493 & 17 & 21 \\
\hline Republica Checa & 492 & 17 & 21 \\
\hline Italia & 487 & 19 & 24 \\
\hline Alemania & 484 & 21 & 25 \\
\hline Liechtenstein & 483 & 20 & 26 \\
\hline Hungría & 480 & 21 & 26 \\
\hline Polonia & 479 & 21 & 27 \\
\hline Grecia & 474 & 23 & 28 \\
\hline Portugal & 470 & 24 & 28 \\
\hline Federación Rusa & 462 & 27 & 29 \\
\hline Latvia & 458 & 27 & 30 \\
\hline Luxemburgo & 441 & 30 & 32 \\
\hline México & 422 & 31 & 14 \\
\hline Brasil & 396 & 32 & 29 \\
\hline Holanda & -- & & 29 \\
\hline & & 2 & 29 \\
\hline
\end{tabular}

Fuente: OCDE. Conocimientos y Destrezas para la vida: Primeros Resultados del Proyecto PISA 2000. Resumen de resultados 2001. p. 16

Como se puede concluir de este estudio auspiciado por la OCDE, la lectura representa hoy día uno de los problemas más importantes a resolver en el contexto internacional, dado que aún en los países clasificados como de alto desarrollo, esta problemática está presente en el contexto de sus sociedades, tal

\footnotetext{
${ }^{5}$ Ibidem.
} 
es el caso de España por ejemplo, donde se ha revelado que el $51 \%$ de la población no es afecta a la lectura, otro país altamente desarrollado donde está vigente esta misma problemática es los Estados Unidos de Norteamérica, donde estudios recientes ponen de manifiesto que "Más de una tercera parte de la población norteamericana tiene problemas de lectura, a tal grado que se estima que 60 millones de norteamericanos son analfabetos funcionales. De acuerdo a un informe confidencial presentado conjuntamente entre the U.S. Department of Education and the U.S. Department of Labor se advierte que en el año 2000, los norteamericanos en edad de trabajar no podrán competir en el mercado por carecer de las habilidades básicas de la lectura y de la escritura.

Otro dato revelador de esta problemática de la sociedad norteamericana señala que las puntuaciones de comprensión lectora, sobre todo las habilidades de interpretar y sintetizar muestran una disminución notable entre los estudiantes de todos los niveles educativos, lo mismo sucede con el tiempo que los estudiantes le dedican a la lectura, que se ha visto reducido en más de un $6 \%$ cada año durante un día normal en todos los niveles educativos",

En el caso de los países menos desarrollados que pertenecen a la OCDE, la problemática de la lectura es aún más grave, tal como lo revelan las conclusiones del Estudio PISA 2000, realizado recientemente por este organismo internacional y que nos muestra que 13 países miembros entre los que destacan México, Luxemburgo, Latvia, Rusia, Portugal, Grecia, Polonia, Hungría, Liechtenstein, Italia y la República Checa, presentaron índicesde lectura significativamente muy por debajo del promedio estadístico de la OCDE en habilidades de lectura.

La UNESCO por su parte al abordar la problemática mundial de la lectura, ha señalado que " Los libros y el acto de leer constituyen los pilares de la educación y la difusión del conocimiento, la democratización de la cultura y la superación individual y colectiva de los seres humanos. En esta perspectiva señala la UNESCO, los libros y la lectura son y seguirán siendo con fundamentada razón, instrumentos indispensables para conservar y transmitir el tesoro cultural de la humanidad, pues al contribuir de tantas maneras al desarrollo, se convierten en agentes activos del progreso. En esta visión, la UNESCO reconoce que saber leer y escribir constituye una capacidad necesaria en si misma, y es la base de otras aptitudes vitales..." 7

A fin de contar con una mayor información confiable sobre la problemática lectora a nivel mundial y poder revertir esta tendencia a mediano y largo plazo, la UNESCO ha realizado por su parte diversas investigaciones al respecto entre sus países miembros. Estos estudios han demostrado que Japón tiene el primer lugar mundial con el $91 \%$ de la población que ha desarrollado el hábito de la lectura, seguido por Alemania con un $67 \%$ y Corea con un $65 \%$ de su población que tiene hábitos de lectura." 8

En lo que respecta a los países más atrasados en hábitos de lectura, México ocupa el penúltimo lugar mundial, de 108 países evaluados con un promedio de $2 \%$ de la población que cuenta con hábitos permanentes de lectura.

\footnotetext{
${ }^{6}$ Judith Mece. Desarrollo del niño y del adolescente : Compendio para educadores. México : SEP : Mcgraw-Hill, 2000. pp. 247-248

${ }^{7}$ UNESCO. Informe sobre la educación en el mundo 2000. Madrid : UNESCO : Santillana, 2000. p. 183

${ }^{8}$ Carlos Paul y Ángel Vargas. "México inmerso en el analfabetismo funcional, una catástrofe silenciosa" En la Jornada (Ene. 15)), 2001.pp. 1-2
} 
A nivel de América Latina y el Caribe, diversos estudios realizados entre 1998 y 1999 y publicados en el año 2000, dan a conocer cual es la situación de las habilidades lectoras de los estudiantes de educación básica en esta región del mundo. Dichas investigaciones alertan sobre el estado crítico en que se encuentran millones de estudiantes latinoamericanos y caribeños en materia de lectura. De 13 países que participaron en estas investigaciones se concluye que con excepción de Cuba país que cuenta con los porcentajes más altos de lectura en sus estudiantes de nivel básico, los 12 países restantes presentan bajos niveles generalizados de lectura entre sus estudiantes.

A este panorama desolador se suman también otros estudios que revelan que "En países como Colombia, Venezuela, Chile, Argentina, Brasil y Ecuador los índices de lectura en la población en general han disminuido drásticamente en los años recientes; en Colombia por ejemplo, de acuerdo a una reciente encuesta nacional, el $40 \%$ de los colombianos manifestaron que no leen libros por falta de hábitos, otro $22 \%$ externo que no lee por falta de tiempo y dinero para comprar libros. Venezuela por su parte reconoce que si se compararan los capacidades lectoras de sus estudiantes con sus similares de Finlandia o de los Estados Unidos, un $90 \%$ de los jóvenes venezolanos quedarían muy por debajo de las capacidades de lectura adquiridas por los Finlandeses y los Norteamericanos en la actualidad. ${ }^{9}$

A continuación se presenta un cuadro comparativo que nos revela la situación lectora de los alumnos de tercer y cuarto grado de educación básica en América Latina y el Caribe que fue publicado en el Primer Estudio Internacional Comparativo sobre Lenguaje, Matemáticas y Factores Asociados, para Alumnos del Tercer y Cuarto Grado de la Educación Básica que se realizó en 1998 bajo el patrocinio de la UNESCO.

\begin{tabular}{|c|c|c|c|c|c|c|}
\hline \multirow[t]{2}{*}{ PAIS } & \multirow[t]{2}{*}{ MEDIAS } & \multicolumn{5}{|c|}{ TÓPICOS EVALUADOS } \\
\hline & & $\begin{array}{l}\text { Identificar } \\
\text { tipos de } \\
\text { textos }\end{array}$ & $\begin{array}{c}\text { Distinguir } \\
\text { emisor y } \\
\text { destinatario de } \\
\text { un texto }\end{array}$ & $\begin{array}{l}\text { Identificar } \\
\text { mensaje de } \\
\text { un texto }\end{array}$ & $\begin{array}{l}\text { Reconocer } \\
\text { información } \\
\text { especifica de } \\
\text { un texto }\end{array}$ & $\begin{array}{l}\text { Identificar } \\
\text { vocabulario } \\
\text { relacionado } \\
\text { con el sentido } \\
\text { de un texto }\end{array}$ \\
\hline Argentina & 277 & 1 & 4 & 4 & 3 & 2 \\
\hline Bolivia & 244 & 4 & 4 & 2 & 2 & 4 \\
\hline Brasil & 269 & 5 & 2 & 1 & 2 & 2 \\
\hline Chile & 272 & 4 & 4 & 3 & 2 & 3 \\
\hline Colombia & 253 & 4 & 5 & 2 & 2 & 3 \\
\hline Cuba & 342 & 3 & 4 & 2 & 3 & 4 \\
\hline Honduras & 230 & 4 & 4 & 4 & 2 & 2 \\
\hline México & 250 & 2 & 5 & 3 & 2 & 4 \\
\hline Paraguay & 250 & 1 & 4 & 2 & 4 & 3 \\
\hline $\begin{array}{l}\text { Rep. } \\
\text { Dominicana }\end{array}$ & 233 & 4 & 4 & 4 & 2 & 2 \\
\hline Venezuela & 242 & 2 & 2 & 4 & 4 & 2 \\
\hline Región & 261 & 2 & 1 & 4 & 4 & 2 \\
\hline
\end{tabular}

FUENTE: UNESCO. Laboratorio Latinoamericano de Evaluación de Calidad de la Educación. Primer Estudio Internacional comparativo sobre Lenguaje, Matemáticas y factores asociados, para alumnos del Tercero y Cuarto grado de educación Básica. P.27

\footnotetext{
${ }^{9}$ Mabel Silva Silva. "Expertos estudian lectoría" En Noticias en el Universal. Caracas : El Universal, 2002. p. 2
} 


\section{LA LECTURA EN EL CONTEXTO MEXICANO}

Antes de analizar cual es la problemáticavigente sobre la lectura en nuestro país, es importante señalar algunos datos estadísticos referenciales sobre la población mexicana, incluyendo el ámbito educativo, pues la problemática lectora incide de manera directa en el desarrollo humano y la calidad de vida de los mexicanos y por ende, en la calidad de la educación que reciben millones de niños y jóvenes estudiantes en el sistema educativo nacional.

De acuerdo a las cifras difundidas por el Instituto Nacional de Geografía y Estadística, la población de México al año 2000 estaba conformada por 97,483,412 habitantes, de los cuales 72,759,822 viven en áreas urbanas y $24,732,590$ habitan en zonas rurales. Cabe destacar además, que del total de la población $53,700,000$ mexicanos se encuentran en situación de pobreza.

En lo referente al sistema educativo mexicano, de acuerdo a las cifras difundidas por la Secretaría de Educación Pública, para el ciclo educativo 2000-2001, la matrícula total del sistema registraba $29,669,046$ estudiantes en todos sus niveles educativos. ${ }^{10}$

Otro dato importante que es digno de citarse en este rubro esel que señaló recientemente el presidente de la república Vicente Fox Quesada en el sentido de que en nuestro país "Existen 32 millones de jóvenes y adultos que no saben leer y escribir, o cuyos niveles de estudio han sido mínimos " 11

A este panorama sombrío se suma la problemática de quienes estando en posibilidades de leer y escribir se comportan como analfabetos funcionales.

Al respecto, como se puede apreciar en los diferentes cuadros comparativos difundidos por la OCDE y la UNESCO, sobre el panorama de la lectura en el mundo, México ocupa los últimos lugares en esta materia en el contexto internacional y regional. Este grave rezago cultural ha sido reconocida ya explícitamente desde el sexenio pasado por el expresidente Ernesto Zedillo Ponce de León y por el actual mandatario mexicano Vicente Fox Quesada, así como por las diversas instituciones educativas y culturales de la nación.

Al hacer referencia a esta situación, el gobierno mexicano ha externado que " La indiferencia de los mexicanos ante la lectura de calidad mantiene al país inmerso en una progresiva catástrofe silenciosa, que de acuerdo a las últimas cifras difundidas por la UNESCO, México ocupa el penúltimo lugar en hábitos de lectura de una lista conformada por 108 naciones del mundo, con un promedio de lectura de 2.8 libros anuales por habitante, cifra muy alejada de los 25 volúmenes recomendados por este organismo internacional, y del promedio de lectura de la sociedad Japonesa, Noruega, Finlandesa y Canadiense que ocupan los primeros lugares a nivel mundial con 47 títulos per cápita." ${ }^{12}$

Por su parte la Secretaria de Educación Pública ha reconocido que "A pesar de lo mucho que ha avanzado la cobertura de la educación básica y el promedio de escolaridad de la población de 15 años, que ya es de 7.7 grados, para la mayoría de los mexicanos la afición de leer libros no es todavía una costumbre, esta falta de lectura de libros entre la población no solamente alfabetizada, sino incluso con muchos años

\footnotetext{
${ }^{10}$ INEGI. Estadísticas sociodemográficas [en línea]. México : INEGI, 2002. Disponible en Internet en: http://www.inegi.gob.mx/estadistica.

11 “Hay 6 millones de analfabetas en México : Fox” En Novedades de México [en línea] . México : Novedades (Ago. 30 ), 2002. Disponible en Internet en http://www.novedades.com.mx

12 Marcos Miranda Gil. Viaje alrededor de la lectura [en línea]. México : SEP, 2000. Disponible en Internet en: http://www.sepic.mx/letras/viaje.htlm.
} 
de escuela, se ha ido convirtiendo en un lastre cada día más pesado, en un factor de atraso cada vez más evidente e incómodo..."13

Como podemos concluir de este breve análisis, la crisis de lectores que vive hoy la sociedad mexicana, amenaza seriamente nuestro proceso educativo y cultural, muy específicamente el desarrollo de nuestros estudiante mexicanos que como lo han demostrado los estudios internacionales y regionales difundidos recientemente, al carecer de las capacidades lectoras no se benefician suficientemente de las oportunidades educativas y no están adquiriendo los conocimientos y habilidades necesarias para tener éxito en sus futuras carreras. Sin una capacidad lectora plenamente desarrollada, nuestros estudiantes no alcanzan un nivel básico de eficiencia, pues fallan en demostrar rutinariamente habilidades y conocimientos que les permitan afrontar retos del futuro, así como en analizar, razonar y comunicar ideas de manera efectiva y en su capacidad para seguir aprendiendo a lo largo de su vida.

\section{LA IMPORTANCIA DE LA LECTURA Y SU PROBLEMÁTICA EN EL CONTEXTO DE LA EDUCACIÓN SUPERIOR EN MÉXICO}

En el contexto de la educación superior mexicana, la problemática lectora se hace presente también en una gran mayoría de los estudiantes universitarios, así lo establece un estudio de La Asociación Nacional de Universidades e Instituciones de Educación Superior (ANUIES)realizado bajo la coordinación de Adrián de Garay Sánchez con el objeto de conocer quiénes son y qué hacen los estudiantes en su tránsito por la educación superior en el nivel licenciatura, tanto dentro como fuera del espacio universitario. Deuna muestra nacional que comprendió a cerca de 10 mil estudiantes de diversas instituciones de educación superior del país, esta investigación llegó a las siguientes conclusiones:

- Un $48.4 \%$ de los universitarios mexicanos dedica entre una y cinco horas a la semana a la lectura de textos escolares y un $21.7 \%$ más dedica entre cinco y diez horas semanales a esta misma actividad. Dicho promedio semanal señala la ANUIES resulta insuficiente para leer los textos necesarios que se incluyen en el currículum universitario y que requieren de un mínimo de lectura de 13 horas a la semana. Para respaldar esta aseveración la Facultad de Psicología de la Universidad Nacional Autónoma de México (UNAM), ha señalado que cualquier estudiante universitario estándar necesita invertir $3 \quad \frac{1}{2}$ horas al día a la lectura de textos para poder cumplir satisfactoriamente con sus tareas y responsabilidades académicas ${ }^{14}$

- Otro indicador importante de este estudio revela que para poder satisfacer sus necesidades de información y lectura, el $50.6 \%$ de los estudiantes universitarios asiste a las bibliotecas de sus escuelas o facultades y raramente visitan otras bibliotecas fuera de su campus universitario. Estos porcentajes nos muestran claramente que la costumbre de visitar la biblioteca universitaria es una práctica poco socorrida por los universitarios hoy en día a pesar de que un $53.5 \%$ opinó que los servicios que se proporcionan en sus bibliotecas son relativamente buenos.

\footnotetext{
13 "Revista Cultural” En reforma. México : Reforma (Sep. 17), 2000. p. 7

${ }^{14}$ Serafín J. Mercado y otros. La lectura de los estudiantes de la Facultad de Psicología de la Universidad Nacional Autónoma de México, medida con un instrumento integrado de velocidad, comprensión y habilidad gramatical. México: Los autores, 1998. p. 9
} 
- Un fenómeno que sin duda esta presente en la comunidad universitaria de México es el hecho de que este estudio reveló que el $86.3 \%$ de los estudiantes universitarios de nuestro país sacan fotocopias para cumplir con la obligación de leer y hacer sus tareas e investigaciones académicas, mientras el $40.7 \%$ declaró que utilizan frecuentemente Internet para satisfacer sus necesidades de información. Estos datos reafirman la denuncia del Centro Mexicano de Protección y Fomento a los Derechos de Autor en el sentido de que "En México se fotocopian 300 millones de libros al año lo que equivale a fotocopiar entre 5 mil y 7 mil millones de páginas, esto ubica a nuestro país, como el número uno de lberoaméricaen materia de piratería de libros" ${ }^{15}$

- En lo que respecta a la compra de libros y revistas especializadas, el estudio de la ANUIES establece que un $11 \%$ de estudiantes universitarios compra estos materiales bibliográficos frecuentemente y un $46.4 \%$ reconoció que casi nunca adquieren libros y revistas para sus estudios. Estos datos señalan categóricamente que los estudiantes universitarios mexicanos no son afectos a la compra de libros y revistas para coadyuvar a su óptimo desarrollo académico.

- En relación al tipo de materiales elaborados como producto de sus lecturas, el $83.6 \%$ de los universitarios reconoció que les sirvió para elaborar resúmenes, un $21.5 \%$ señaló que gracias a la lectura les era posible elaborar fichas de trabajo, y un 34\% manifestó que la lectura les facilitó la elaboración de esquemas de trabajo. Estos datosnos demuestran que la lectura de libros y revistas especializadas constituye una práctica necesaria para que los estudiantes preparen sus clases y realicen sus trabajos escolares de manera óptima, lo que sin duda permite distinguir a los lectores universitarios más asiduos por su disposición, mejor vocabulario, más acervo informativo y por una gran habilidad y capacidad de síntesis, reflexión y análisis.

- Otro elemento importante que nos proporcionó esta investigación de la ANUIES, es el hecho de que una gran mayoría de los estudiantes universitarios mexicanos carecen de habilidades y motivaciones para leer y realizar trabajos en grupo. De acuerdo a estos resultados solamente un $13.5 \%$ manifestó que con frecuencia leían y hacían trabajos en equipo, mientras que un 78.5 \% externó que nunca o casi nunca han leído en grupo para hacer sus trabajos académicos posteriormente.

- En cuanto a su participación en clase como consecuencia de sus lecturas previas, el $54,2 \%$ de los universitarios mexicanos respondieron que casi siempre les fue de utilidad para exponer en clase, un $50.6 \%$ señaló que la lectura les proporcionó elementos suficientes para participar casi siempre en clase y un $46.3 \% \%$ señaló que la lectura les brindó la confianza para preguntar en clase a fin de enriquecer sus conocimientos y de poder discutir con regularidad los puntos de vista de sus profesores. Como se puede apreciar de estos porcentajes, un $50 \%$ en promedio de los estudiantes universitarios consideraronque la lectura les brindó un apoyo importante para estar activos y participar en sus clases cotidianamente.

\footnotetext{
${ }^{15}$ Sergio Raúl López. "Fotocopian al año 300 millones de libros". En Reforma [en línea]. México : Reforma (Sep. 9), 2002. Disponible en Internet en http:// www.reforma.com
} 
- Otro indicador importante que nos reveló este estudio de la ANUIES sobre el hacer de los estudiantes universitarios, es el hecho de que un 29.3\%no cuenta con enciclopedias en su casa y otro 38.7 \%reconoció que carecían de libros especializados para realizar sus lecturas y tareas académicas en sus hogares. Estos datos ponen de manifiesto de maneraobjetiva que en una gran mayoría de los hogares mexicanos, por diversas circunstancias, se carece de una cultura en favor de la lectura en el contexto familiar.

- Estos datos difundidos por la ANUIES nos muestran claramente la problemática de la lectura que presentan hoy en día miles de estudiantes universitarios matriculados en las diversas Instituciones de Educación Superior de nuestro país, derivada principalmente de la falta de hábitos, por la poca motivación que reciben de sus maestros y del nulo interés de sus "Alma Mater" por fortalecerles a través de programas permanentes de fomento a la lectura, el desarrollo de sus capacidades lectoras en el entendido de que los paradigmas vigentes en el contexto mundial, exigen de los nuevos modelos de formación superior universitaria del desarrollo de nuevas características en el perfil profesional de sus egresados en los umbrales del siglo XXI en el contexto de la sociedad del conocimiento.

\section{LA IMPORTANCIA Y LA PROBLEMÁTICA DE LA LECTURA EN LAUNIVERSIDAD JUÁREZ AUTÓNOMA DE TABASCO (UJAT)}

La Universidad Juárez Autónoma de Tabasco (México) tiene una matrícula total de 28,082 estudiantes y 1,659 docentes distribuidos en nueve Divisiones Académicas y una Extensión de los Ríos. La matrícula estudiantil está distribuida de la siguiente manera:

\section{Nivel Técnico 491 estudiantes}

Licenciatura 22,265 estudiantes

\section{Especialidad129 estudiantes}

Maestría 340 estudiantes

\section{Centros de extensión 4,857 estudiantes}

Actualmente la UJAT oferta una opción a nivel técnico, 33 licenciaturas, 18 especialidades, 14 Maestrías, un doctorado recientemente iniciado, así como 12 talleres culturales, 16 talleres deportivos, 6 cursos de lenguas extranjeras y un curso para profesor de idiomas. Entre su infraestructura académica sobresale su sistema bibliotecario integrado por ocho bibliotecas universitarias y una biblioteca pública con características históricas. Entre los objetivos del sistema bibliotecario se encuentran la prestación óptima de servicios bibliotecarios y el desarrollo continuo de actividades de fomento a la lectura a fin de coadyuvar a la excelencia académica, el desarrollo de la investigación y el impulso a la vinculación de la comunidad universitaria con la cultura y las artes. ${ }^{16}$

Atenta a los nuevos escenarios mundiales y ante las exigencias de los paradigmas vigentes en el campo educativo, la UJAT ha reconocido puntualmente que "... El dinamismo internacional y nacional que caracteriza a nuestro tiempo, impele a establecer métodos renovados de educación y procedimientos para su aplicación, en este contexto, la educación superior tiene hoy un gran valor estratégico en el impulso de 
las transformaciones que el desarrollo del país exigen... Por ello, se hace imprescindible formar hombres y mujeres que a partir de la comprensión de nuestros problemas sean capaces de formular soluciones que contribuyan al progreso de la nación; elevar la calidad del personal docente; fortalecer la cultura de la investigación e impulsar vigorosamente la participación de la comunidad universitaria en todas las actividades culturales que se promuevan en el interior y el exterior de la universidad..." 17

A fin de hacer posible esta visión, la UJAT ha reconocido en primerainstancia que entre sus debilidades académicas se encuentra el hecho de que "En el caso específico de sus estudiantes de manera muy general puede decirse que existen en ellos escasos o nulos hábitos de lectura, dificultades para comprender y analizar textos, pobre información acerca de los acontecimientos locales, nacionales y mundiales, desconocimiento de métodos de lectura y estudio, poco interés hacia la investigación bibliográfica y documental, mala ortografía y desconocimiento de los principios básicos de redacción..." ${ }^{18}$

Esta problemática es reafirmada por diversas investigaciones y trabajos de tesis desarrolladas en el ámbito de esta universidad mismas que han sido presentadas entre 1995 y 1999 y donde se puede establecer que :

Una mayoría de los estudiantes universitarios de la UJAT carecen de las capacidades lectoras necesarias para mejorar la calidad de sus aprendizajes. Debido a estas deficiencias, presentan diversos problemas académicos que se traducen en : Bajos índices de participación en clases, deficiencias en el desarrollo de sus investigaciones escolares, adquisición de un escaso vocabulario, menor dominio de la expresión oral y escrita, poca información sobre el acontecer mundial, nacional y estatal, bajo aprovechamiento de los beneficios que les proporciona el sistema bibliotecario, desmotivación al considerar el acto de leer como una valoración negativa de obligatoriedad, además de que están inmersos en una cultura de la fotocopia en forma de antologías y compilaciones de lecturas fragmentadas (que muchas veces no llevan mayores referencias bibliográfica sobre las fuentes originales de las que fueron fotocopiadas) obstaculizando con ello, la consulta de los textos originales en las bibliotecas universitarias divisionales.

Ante esta crisis lectoraen la que se encuentra inmersa la comunidad universitaria de la UJAT, cabe preguntarse ¿Qué se puede hacer para desarrollar las capacidades de lectura en los estudiantes universitarios de la UJAT?

La solución no es simple, pues como bien lo ha señalado el destacado escritor y promotor de la lectura Felipe Garrido, "Los lectores no nacen, se hacen", y para que los estudiantes de la UJAT se conviertan en excelentes lectores en el menor tiempo posible, se tiene que poner en práctica de manera inmediata un Programa Estratégico Universitario de Fomento a la Lectura, a fin de hacer de los estudiantes tabasqueños mejores universitarios a través de la lectura en los próximos años.

\section{CONCLUSIONES}

- Es importante dejar claro en este artículo que actualmente, la lectura empieza a ser reconocida ya a nivel mundial, como un elemento fundamental y estratégico en el desarrollo de las naciones y en el bienestar de los ciudadanos.

\footnotetext{
${ }^{16}$ Universidad Juárez Autónoma de Tabasco. Anuario Estadístico, 2001. Villahermosa, Tab. : UJAT, 2001.pp. 2-5

${ }^{17}$ Universidad Juárez Autónoma de Tabasco." Excelencia académica, Compromiso social”: Programa de trabajo, 20002004. México : UJAT, 2000.pp. 27-30
} 
- En estas nuevas circunstancias, la lectura debe ser considerada hoy en día como un proceso interactivo de comunicación donde se establece una relación entre el texto y el lector, quien a través de su capacidad lectora; procesa, organiza, sintetiza, analiza y valora la información leída para interiorizarla como lenguaje personal construyendo su propio significado.

- En el contexto de los fenómenos de la globalización, la regionalización, el desarrollo tecnológico y la sociedad de la información, la educación superior y la lectura se constituyen en un binomio determinante en el desarrollo económico y social de las naciones.

- En este concierto, los universitarios deben de fortalecer sus capacidades de lectura a lo largo de la vida para apropiarse de los conocimientos a fin de tener una efectiva participación en la sociedad moderna para enfrentarse con éxito a los retos del futuro.

- A pesar del reconocimiento mundial que se le ha dado a la lectura en el desarrollo educativo y cultural de los individuos, y en el progreso de las naciones, actualmente existe un grave rezago de programas de fomento a la lectura en diversos países desarrollados y subdesarrollados especialmente en el contexto educativo universitario.

- Dentro de esta problemática, México se ubica en los últimos lugares a nivel regional y mundial.

- Dado la magnitud de esta problemática, especialmente entre los estudiantes universitarios, diversas autoridades e instituciones han reconocido que la falta de lectura de los mexicanos amenaza seriamente el desarrollo educativo y cultural de nuestro país, por lo que deben de emprenderse diversas acciones para resolver esta crisis de lectores.

- En el caso específico de la UJAT, diversos estudios e investigaciones han revelado que los universitarios tabasqueños carecen de capacidades lectoras básicas que afectan su formación académica y profesional, por lo que las autoridades universitarias deben de implementar de manera inmediata un Programa Estratégico de Fomento a la Lectura a fin de transformar en el corto y mediano plazo a los estudiantes universitarios de la UJAT en mejores lectores.

\section{BIBLIOGRAFÍA}

ARENZANA, Ana y Aureliano García, Espacios de lectura : estrategias metodológicas para la formación de lectores. México : FONCA, 1995. 175 p. "El Ilano sigue en llamas" en Expansión. (Sep.) México: Expansión, 1996. 152 p.

FERNÁNDEZ SANTILLÁN, Nancy Ileana y otros. Análisis estadístico : Saber leer, factor determinante en el rendimiento académico. [en línea] .La Laguna, Coahuila : Los autores, 2002. Disponible en Internet en http://www.ciiadet.edu.mx.

GÓMEZ PALACIOS, Margarita y otros. La lectura en la escuela. México : SEP, 1996. 311 p.

INEGI. Estadísticas sociodemográficas. [en línea]. México : INEGI, 2002. Disponible en Internet en: http://www.inegi.gob.mx/estadística. "Hay 6 millones de analfabetas en México : Fox." En Novedades México (Ago. 30) [en línea]. México : Novedades, 2002. Disponible en Internet en http://www.novedades.com.mx

\footnotetext{
${ }^{18}$ Universidad Juárez Autónoma de Tabasco. Plan de Desarrollo Institucional, 1996-2000. México : UJAT, 1996. p. 47
} 
LÓPEZ, Sergio Raúl. "Fotocopian al año 300 millones de libros”. en Reforma (Sep. 9) [en línea]. México :

Reforma, 2002. Disponible en Internet en http:// www.reforma.com

MEECE, Judith. Desarrollo del niño y del adolescente : Compendio para educadores. México : SEP :

McGraw-Hill, 2000. 265 p.

MERCADO, Serafín J., y otros. La lectura de los estudiantes de la Facultad de Psicología de la Universidad Nacional Autónoma de México, medida con un instrumento integrado de velocidad, comprensión y habilidad gramatical. México : Los autores, 1998. 45 p.

MIRANDA GIL, Marcos. Viaje alrededor de la lectura [en línea]. México, 2002. Disponible en Internet http://www.sepic.mx/letras/viaje.html. OCDE. Conocimientos y destrezas para la vida: Primeros resultados del proyecto PISA. Educación y destrezas. Madrid : OCDE : Ministerio de Educación, Cultura y Deporte,2001. $31 \mathrm{p}$.

OECD. Programme for International student Assessment. Reading, mathematical and scientific literacy. París : OECD, 2000. 159 p. "Revista Cultural" En Reforma. México : Reforma, (Año 7, 2,568) (Sep. 17), 2000. $129 \mathrm{p}$.

RUIZ, Elisa. "La esencia de la lectura no es obtener información, sino vivir una experiencia" en Rumbo Nuevo. (No. 12,879) (Mar. 3). Villahermosa, Tab.: Rumbo Nuevo, 2002. p. v.

SILVA, SILVA, Mabel. "Expertos estudian lectoría"en Noticias en El Universal.

Caracas : El Universal, 2002. 2 p. UNESCO. Informe sobre la educación en el mundo 2000.

Madrid : UNESCO : Santillana, 2000. 235 p.

UNESCO. World Education report 2000 : The right to education : towars education forall throughout life. París : UNESCO Publishing, 2000. 178 p.

UNESCO. OREALC. Primer Estudio Internacional Comparativo sobre lenguaje, matemática y factores asociados, para alumnos del tercer y cuarto grado de la educación básica. Segundo informe. Santiago de Chile : UNESCO, 2000. $48 p$

UNIVERSIDAD JUÁREZ AUTÓNOMA DE TABASCO. Anuario estadístico, 2001. Villahermosa, Tab. : UJAT, 2001. $73 \mathrm{p}$.

UNIVERSIDAD JUÁREZ AUTÓNOMA DE TABASCO. Autodiagnósticos de las Divisiones Académicas y la extensión de los Ríos. Villahermosa, Tab. : UJAT, 2001. 10 v.

UNIVERSIDAD JUÁREZ AUTÓNOMA DE TABASCO Excelencia académica, compromiso socia I: Programa de trabajo 2000-2004. Villahermosa, Tab.,: UJAT, 2000. 81 p.

VARGAS, Paul Carlos y Ángel. "México inmerso en el analfabetismo funcional, una catástrofe silenciosa" en La Jornada (Ene. 15), 2000. México : La Jornada, 2000. p. v. 